untersucht. Den Abschluß des Bandes bildet ein Manuskript aus dem 9. islamischen Jahrhundert, dem wegen seiner vielfältigen (biographischen und literarischen) Angaben ebenfalls besondere Bedeutung im Rahmen der malikitischen Uberlieferungs- und Quellengeschichte zukommt.

Wenn auch der Band für den größten Teil der Leser dieser Zeitschrift nicht von unmittelbarem Interesse sein mag, so ist er doch bemerkenswert, weil er im Bereich der Grundlagenforschung wertvolle Arbeit leistet, überdies bestehen durchaus Beziehungen zum Themengebiet dieser Zeitschrift: Hauptverbreitungsgebiete der malikitischen Rechtsschule waren Nord-, West- und Zentralafrika (und das muslimische Spanien), wo sie noch in der Kolonialzeit das endogene Rechtssystem bildete, das Gegenstand von "Modernisierungsbestrebungen" seitens der Kolonialmächte war.

Ulrich Deffaa

\title{
Keith Hodkinson
}

\section{Muslim Family Law: A Sourcebook}

London, Canberra: Croom Helm, 1984, 401 S., $\mathfrak{E} 14.95$ (mit Auswahlbibliographie, Glossar und Index).

Keith Hodkinson behandelt in seinem Buch "Muslim Family Law: A Sourcebook" das Familienrecht der hanafitischen Rechtsschule, wie es in Indien und Pakistan in Gestalt des sog. "Anglo-Muhammadan Law" heute von den Gerichten angewendet wird. Das Buch ist in erster Linie als Studienbuch für Studenten des islamischen Rechts in Großbritannien gedacht und soll die Lücke schließen, die hinsichtlich der Zugänglichkeit von Primärquellen, also Gesetzestexten und (insbesondere aktueller) Rechtsprechung aus Indien und Pakistan besteht. Die fünf Kapitel des Buches setzen sich jeweils aus einer knappen Darstellung der rechtlichen Situation unter Einbeziehung der einschlägigen Gesetzestexte und einem ausführlichen Rechtsprechungsteil, in dem Gerichtsentscheidungen im Wortlaut reproduziert sind, zusammen. Die wiedergegebenen Gesetze und Entscheidungen sind am Ende des Buches aufgelistet. Ein Glossar arabischer Begriffe, ein Index und eine auf englischsprachige Titel beschränkte und nach Sachgebieten gegliederte Auswahlbibliographie sind ebenfalls beigefügt. Berücksichtigung fand Material, soweit es dem Autor bis August 1982 vorlag.

Im einleitenden ersten Kapitel (Text S. 1-26, Rechtsprechungsteil S. 27-87) erläutert Hodkinson kurz die Natur des islamischen Rechts, seine Hauptcharakteristika und seine Quellen und gibt einen Uberblick über die orthodoxen (sunnitischen) und nicht-orthodoxen (shiitischen) Rechtsschulen unter Hinweis auf ihre Hauptunterschiede. Das sog. "Anglo-Muhammadan Law" Indiens und Pakistans weist die Besonderheit auf, daß islamisches Recht von britischen Kolonialgerichten angewendet wurde und so unter dem Einfluß englischen Rechtsdenkens sowohl in seiner Substanz als auch in seinen formalen 
Quellen deutliche Unterschiede zum klassischen islamischen Recht entwickelt hat. Während Indien und Pakistan, als damaliges Britisch-Indien, bis zu ihrer Unabhängigkeit und Teilung im Jahr 1947 die gleiche koloniale Rechtsgeschichte miteinander verband, nahm seitdem, aufgrund der unterschiedlichen Staatsauffassungen in der islamischen Republik Pakistan und in der säkularen Republik Indien, das islamische Recht in beiden Staaten eine unterschiedliche Entwicklung. In Pakistan, wo etwa 97 \% der Bevölkerung Muslims sind und der Islam die Staatsreligion bildet, müssen laut Verfassung alle Gesetze im Einklang mit Koran und Sunna stehen. In Indien, wo die Muslims mit etwa $12 \%$ lediglich eine Minderheit darstellen, sieht die Verfassung zwar vor, daß zunächst das bestehende Recht, einschließlich des islamischen, fortgilt; jedoch unterliegt auch das islamische persönliche Recht der Gesetzgebungskompetenz des Staates (teilweise strittig, vgl. Art. 25, 26 der Verfassung) und es besteht ein Verfassungsauftrag zum Erlaß eines allgemeinen Zivilgesetzes, der jedoch bisher politisch nicht durchsetzbar war. Entsprechend ist auch die Anwendbarkeit islamischen Rechts in Pakistan in weiterem Umfang vorgesehen als in Indien. Die in Pakistan im Jahr 1961 erlassene Muslim Family Laws Ordinance zeigt nach Hodkinson eine liberal-moderne Ausrichtung, die, trotz des in den 70er Jahren erstarkenden islamischen Fundamentalismus, im Bereich des Familienrechts nach wie vor bestehe. In Indien nehme man mangels entsprechender Reformgesetzgebung in einigen Fragen stärker Bezug auf das klassische hanafitische Recht und zeige damit teilweise eine konservativere Haltung bei der Anwendung islamischen Familienrechts.

Die folgenden Kapitel bringen eine Fülle von Details zum Recht der Eheschließung und Ehescheidung, der Ehewirkungen und der Eltern-Kind-Beziehung. Das zweite Kapitel über die "Gültigkeit der Eheschließung" (Text S. 89-115, Rechtsprechungsteil S. 116-130) befaßt sich zunächst mit der islamischen Auffassung von der Ehe als zivilrechtlichem Vertrag. Im Zusammenhang mit dem Ehemindestalter wird die Kinderheirat behandelt, die zwar in beiden Ländern strafrechtlich verfolgbar ist, an deren zivilrechtlicher Wirksamkeit aber nicht gerüttelt wurde. Die Polygamie, vom Koran lediglich mit der "moralischen" Einschränkung versehen, daß ein Ehemann in der Lage sein muß, alle Ehefrauen gleich zu behandeln, ist in Indien nach wie vor ohne rechtliche Einschränkung zulässig, während Pakistan mit der Muslim Family Laws Ordinance 1961 versuchte, gewisse Restriktionen einzuführen. Die Nichtbeachtung dieser Vorschriften berührt jedoch nicht die Wirksamkeit der Eheschließung. Der Streit um Pro und Contra der Polygamie hat nach Hodkinson für Indien und Pakistan keine große praktische Bedeutung, weil die Zahl polygamer Ehen heute verhältnismäßig gering sei (S. 108). Die komplizierten Probleme, die die gemischt-religiöse Ehe aufwirft, werden erörtert sowie die Ehehindernisse und Formerfordernisse, die Eheschließung durch Vertreter, die Ehevermutung bei Zusammenleben, Fragen der Registrierung und die Folgen materieller und formeller Mängel der Eheschließung. Schließlich wird das shiitische Eherecht mit seiner zeitlich befristeten Ehe (mut'a) und anderen Besonderheiten angesprochen. Das dritte Kapitel (Text S. 131-152, Rechtsprechungsteil S.152-218) hat die rechtlichen Beziehungen zwischen den Ehepartnern zum Gegenstand. Breiten Raum nehmen die 
Ausführungen zum "dower" (mahr/mehr) ein, einer Zahlungspflicht des Ehemanns an die Ehefrau (nicht zu verwechseln mit "dowry", der von der Familie der Braut zu zahlenden Mitgift). Hodkinson erklärt "dower" als eine aus der Eheschließung entstehende Verpflichtung und nicht als Ehegültigkeitsvoraussetzung. Zwei Typen von "dower" sind möglich, das auf Abruf der Ehefrau fällige "prompt dower" und dasjenige, das erst bei Beendigung der Ehe fällig wird ("deferred dower"). Das Recht der Ehefrau auf "dower" ist nach Hodkinson gewissermaßen als Ausgleich dafür zu sehen, daß das islamische Recht der Frau nach Beendigung der Ehe keine Unterhaltsansprüche zubilligt. Auch die erbrechtlich schlechte Position der Witwe könne dadurch aufgebessert werden. Das "dower" erreicht nach Hodkinson of thohe Summen, was den Ehemann von der (kostspieligen) Scheidung abhalten solle, aber häufig auch auf Prestigegründen beruhe. So werden vielfach Scheinvereinbarungen über hohe "dower"-Beträge geschlossen und publik gemacht, während geheime Privatvereinbarungen weit niedrigere Beträge festsetzen. Die Institution des "dower" wurde in beiden Ländern heftig gegen gesetzgeberische Einschränkungen verteidigt. Weitere Gegenstände des dritten Kapitels sind das Recht auf eheliche Lebensgemeinschaft, die Kontrollbefugnisse des Ehemannes über die Ehefrau, die der Ehefrau zustehenden Unterhaltsansprüche sowie deren Dauer und Durchsetzbarkeit, ehevertragliche Vereinbarungen und die Stellung der polygamen Ehefrauen.

Im vierten Kapitel, "Dissolution of Marriage" (Text S. 219-247, Rechtsprechungsteil S. 247-306), wird die ganze Bandbreite von Eheauflösungsmöglichkeiten erörtert. Dies sind der Tod bzw. vermutete Tod eines Ehegatten und die zahlreichen Formen von talaq, der einseitigen Verstoßung, und faskh, der gerichtlichen Eheauflösung. Die jeweiligen Folgen hinsichtlich der Wartefrist idda, Unterhaltsansprüchen der Frauen etc. werden ebenfalls erörtert. Hinsichtlich der Ehescheidung hat staatliches Recht besonders deutlich in das klassische hanafitische Recht eingegriffen, um die Position der Ehefrau zu verbessern. Der Dissolution of Muslim Marriage Act 1939 erweiterte, teilweise unter Einbeziehung malikitischer Lehren, die Scheidungsmöglichkeiten der Ehefrau beträchtlich gegenüber dem hanafitischen Recht.

Die Eltern-Kind-Beziehung ist Gegenstand des fünften Kapitels (Text S. 307-314, Rechtsprechungsteil S. 314-359). Da die Ehelichkeitsvermutungen des auf englischem Recht beruhenden Indian Evidence Act 1872 einerseits und des islamischen Rechts andererseits voneinander differieren, kommt für den Status eines Kindes der Rechtswahl besondere Bedeutung zu. In Pakistan ist islamisches Recht auch für Statusfragen anwendbar, während in Indien eine entsprechende Anwendbarkeitsvorschrift nicht existiert und somit nach Hodkinson die Ehelichkeitsvermutung staatlichen Rechts auch für Muslims gilt. Neben der Ehelichkeitsvermutung kann unter bestimmten Voraussetzungen die Anerkennung des Kindes durch den Ehemann der Mutter die Legitimität des Kindes bewirken. Das nichteheliche Kind hat keine Verwandtschaftsbeziehung zum Vater, der aber nach staatlichem Recht trotzdem unterhaltspflichtig sein kann. Das Rechtsinstitut der Adoption ist dem islamischen Recht unbekannt. Während das Sorgerecht für eheliche Kinder nach hanafitischem Recht für Töchter bis zur Pubertät, für Söhne bis zum Alter von sieben Jahren der Mutter zusteht, danach dem Vater, führten staatliches Recht und 
Rechtsprechung das Kindeswohlprinzip ein. Der Vater ist unterhaltspflichtig für seine legitimen Kinder, aufgrund staatlicher Gesetze nun nicht mehr bis zu ihrer Pubertät bzw. Verheiratung (so islamisches Recht), sondern bis zu ihrer Volljährigkeit (bzw. Verheiratung bei Töchtern).

Hodkinsons Buch enthält reichhaltige Informationen zur Rechtslage in Indien und Pakistan und weist eine sorgfältige Auswertung des klassischen islamischen Rechts der hanafitischen Schule, der staatlichen Gesetzgebung und Rechtsprechung auf. Obwohl der Autor sich überwiegend auf eine deskriptive Wiedergabe beschränkt, was durchaus dem Charakter eines Quellenwerkes entspricht, wird durch die Materialauswahl das Wechselspiel zwischen dem unkodifizierten islamischen Recht und den rechts- und staatspolitisch determinierten Eingriffen der beiden Staaten durch Kodifikationen und Rechtsprechung deutlich. Für Analysen unter reformmethodologischen, ${ }^{1}$ rechtssoziologischen u. a. Gesichtspunkten kann auf die in der Bibliographie angegebenen Werke zurückgegriffen werden. Wohl wegen der Zielsetzung als Studienbuch hat der Autor die Quellen meist nicht diskutiert, sondern es beim reinen Zitieren belassen. Wenn er ab und zu von diesem Prinzip abweicht und Gesetzestexte und Entscheidungen unmittelbar kommentiert (wie z. B. im vierten Kapitel, S. 224 ff.), so weckt dies im Leser allerdings den Wunsch, derartige Anregungen zur Interpretation und zum Verständnis der Texte häufiger zu erhalten.

Das Manko des Buches ist seine Unübersichtlichkeit, was gerade für eine Quellensammlung von großem Nachteil ist. Eigener Text des Autors und Quellentexte sind drucktechnisch nicht voneinander abgehoben und nicht deutlich kenntlich gemacht. Auch ist das Recht von Indien und Pakistan nicht immer ohne weiteres unterscheidbar. Zur Unübersichtlichkeit trägt weiter bei, daß eine Untergliederung innerhalb der Kapitel nur ansatzweise stattfindet und weder mit Ordnungsziffern versehen noch im Uberblick wiedergegeben ist, so daß die Gliederungspunkte erst im Lauf der Lektüre aufzuspüren sind. Nützlich für die Arbeit mit den Quellen wäre es auch, wenn die abgedruckten Gerichtsentscheidungen mit Hinweisen auf die wichtigsten von ihnen behandelten Rechtsprobleme versehen wären. Der Index und das arabische Glossar sind unvollständig, und die Erklärungen der Begriffe sind teilweise wenig hilfreich (z. B. waqf = "settlement"). Dies alles bewirkt, daß die Suche nach bestimmten Aussagen oder Quellen sich mitunter mühsam gestaltet. Es fällt zwar weniger ins Gewicht, wenn man das Buch von vorn bis hinten durchliest, und das hat der Autor vermutlich auch mehr im Auge gehabt. Doch auch als Nachschlagewerk kann es von großem Nutzen sein, und es bleibt zu wünschen, daß bei einer eventuellen Neuauflage das Layout des Buches übersichtlicher neugestaltet wird, damit der unschätzbare Vorteil, die Primärquellen unmittelbar bei der Hand zu haben, besser genutzt werden kann.

Ulrike Wanitzek

1 Vgl. hier insbesondere die in VRU 18 (1985), S. 78 ff. rezensierte Untersuchung Espositos, die ebenfalls das Familienrecht Pakistans zum Gegenstand hat (im Vergleich mit dem ägyptischen Recht). 